\title{
Eniric: Extended NIR Information Content
}

\author{
J.J. Neal ${ }^{1,2}$ and P. Figueira ${ }^{3,1}$ \\ 1 Instituto de Astrofísica e Ciências do Espaço, Universidade do Porto, CAUP, Rua das Estrelas, \\ 4150-762, Porto, Portugal 2 Departamento de Física e Astronomia, Faculdade de Ciências, \\ Universidade do Porto, Rua do Campo Alegre, 4169-007, Porto, Portugal 3 European Southern \\ Observatory, Alonso de Córdova 3107, Vitacura, Casilla 19001, Santiago 19, Chile
}

DOI: $10.21105 /$ joss.01053

\section{Software \\ - Review ๘ \\ - Repository [ד \\ - Archive ${ }^{x}$}

Submitted: 11 September 2018 Published: 08 May 2019

\section{License}

Authors of papers retain copyright and release the work under a Creative Commons Attribution 4.0 International License (CC-BY).
With recent high-precision spectrographs targeting radial velocity (RV) precision at the $10 \mathrm{cms}^{-1}$ level (e.g. F. Pepe et al., 2014) in the quest to find smallest planets, it is important to understand the theoretical precision attainable in stellar spectra. Eniric provides a simple way to calculate the theoretical spectral quality and RV precision (i.e., information content) of synthetic and/or observed stellar spectra given vectors of wavelength and photon flux.

Written in Python 3, Eniric calculates the fundamental photon noise RV precision as formulated in Connes (1985) and F. Bouchy, Pepe, \& Queloz (2001). It is an improved version of the software used in Figueira et al. (2016) for calculating the RV precision of synthetic M-dwarf spectra in the near-infrared (NIR) bands. The code was refactored, with hard-coded constraints removed, making it faster and simpler to explore a larger combination of parameters (e.g. not limited to M-dwarfs and NIR wavelengths).

Eniric contains several independent functions to transform observed and synthetic spectra, such as wavelength selection, broadening, SNR normalization and to compute RV precisions.

Eniric performs rotational and instrumental broadening of spectra through convolution with a rotational kernel (Gray, 2005) and gaussian kernel respectively. Both kernels are wavelength dependant and do not require a uniformly spaced wavelength vector, unlike the convolution functions given in PyAstronomy. Eniric utilizes the embarrassingly parallel nature of the convolutions (each pixel can be calculated independently of its neighbours) to compute the convolutions in parallel; the convolution results are also cached using Joblib to avoid re-computation. This improves the convolution performance but not to the level achievable by algorithms that require an equal wavelength spacing and use fixed kernels (only valid for small wavelength regions), e.g. the "fast" convolutions provided in PyAstronomy.

Eniric enables the relative precision between synthetic spectra by allowing for normalization to a user defined signal-to-noise ratio (SNR) per pixel at a specific wavelength. Although user definable the default choice is a SNR of 100 at the center of the J-band $(1.25 \mu \mathrm{m})$ as used in Figueira et al. (2016).

The precision calculations are not limited to the large spectroscopic bands, but can also be performed on narrow wavelength slices along the entire spectrum. This allows one to explore the RV precision across the entire spectrum and perform comparision between observations and synthetic libraries (e.g. É. Artigau et al., 2018).

Extraneous information not included in the spectra (i.e., not photon noise nor line content information) can be included in the precision calculation through the use of a spectral mask. This mask can be used to indicate which spectral lines are to be included/excluded (via a binary mask) or if some spectral lines should receive more statistical weight for an external reason. For example, masks derived from an atmospheric absorption spectrum can be used to explore the treatment and correction the atmospheric absorption on the 


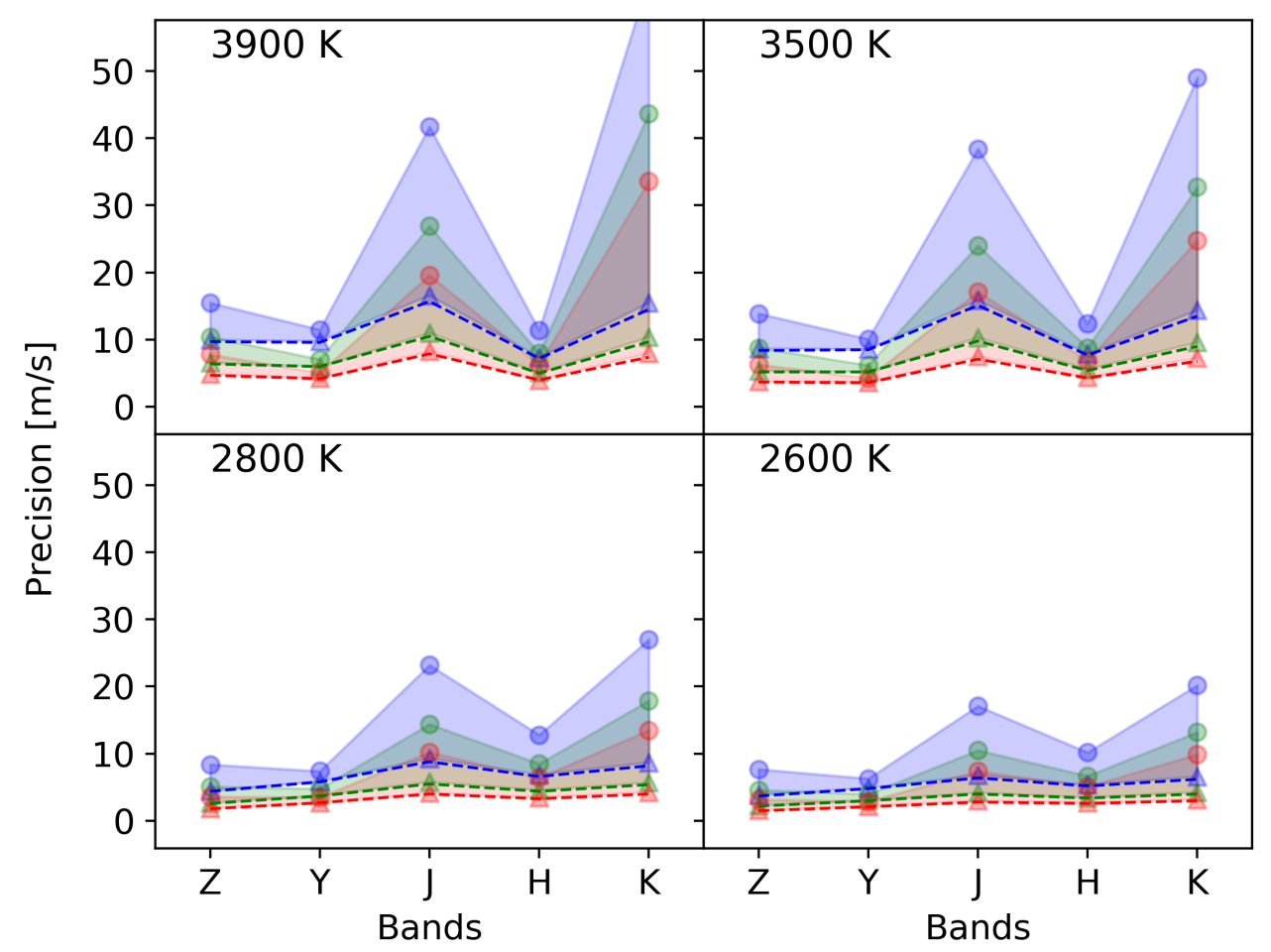

Figure 1: Precision achieved with Eniric as a function of spectral band for stars with a rotational velocity of vsini $=1.0 \mathrm{kms}^{-1}$ and temperatures $3900 \mathrm{~K}, 3500 \mathrm{~K}, 2800 \mathrm{~K}, 2600 \mathrm{~K}$, corresponding to spectral types $M 0, M 3, M 6$, and $M 9$ respectively. The dashed line represents the theoretical limits imposed by the full spectrum, and the filled area represents the values within the limits set by the complete removal of atmospheric absorption regions (circles) and the perfect correction of atmospheric absorption (triangles); blue, green, and red represent the results obtained for resolutions of 60000 , 80000 , and 100000 , respectively. The spectra were normalized to have a SNR of 100 per resolution element as measured at the center of the J-band. This is similar to Figure 1 from (Figueira et al., 2016) but with updated precision values. 
RV precision (Figueira et al., 2016). A typical treatment for the Earths atmosphere is the exclusion (via masking) of any region within $30 \mathrm{kms}^{-1}$ of an atmospheric absorption line deeper than $2 \%$, to account for Earths barycentric motion.

The script phoenix_precision.py is provided to easily compute relative RV precisions for synthetic spectra from the PHOENIX-ACES (Husser et al., 2013) and BT-Settl (CIFIST2011-2015) (Baraffe, Homeier, Allard, \& Chabrier, 2015) libraries. The Grid Tools module from Starfish (Czekala, Andrews, Mandel, Hogg, \& Green, 2015) is used to load in the library spectra given the identifying stellar parameters i.e, temperature, logg, metalicity and alpha. The RV precision is computed under the three separate conditions of Figueira et al. (2016). These are: the full spectrum, the exclusion of regions within $30 \mathrm{kms}^{-1}$ of deep telluric lines (binary mask), and the full spectrum assuming a "perfect" telluric correction in which the spectrum is recovered but with an increased variance dependant on the telluric line depth (weighted mask). The results are tabulated for all combinations of the spectral parameters, SNR, instrument resolutions, rotational velocities, pixel sampling, and wavelength choices provided to the script. An example of relative precision results is shown in Figure 1.

Eniric has been recently used to calculate relative RV precision of M-dwarf spectra for use in the Exposure Time Calculators of NIRPS (F. Bouchy et al., 2017) and SPIRou (É. Artigau et al., 2014), two new high-resolution NIR spectrographs. For SPIRou these were specifically calculated for the instruments resolution $(\mathrm{R}=75,000)$ and for all stellar temperatures between $2500-4000 \mathrm{~K}$. It is also currently being used to compare the theoretical precision between observed CARMENES spectra (A. Reiners et al., 2018) and their synthetic library counterparts.

Eniric is available on GitHub with documentation found in the README.md and at ReadtheDocs with usage examples provided as Jupyter notebooks. It utilizes packages from the scientific Python stack including NumPy (T. E. Oliphant, 2015) and SciPy (Jones, Oliphant, Peterson, \& others, 2001-2001--), Matplotlib (Hunter, 2007), Pandas (McKinney, 2010), and Astropy (Astropy Collaboration et al., 2013; Price-Whelan et al., 2018). It also uses Joblib("Joblib," n.d.), Starfish (Czekala et al., 2015), and tqdm ("Tqdm/tqdm," n.d.).

\section{Acknowledgements}

This work was supported by Fundação para a Ciência e Tecnologia (FCT) (Portugal) research grants through national funds and from FEDER (Fundo Europeu de Desenvolvimento Regional) through COMPETE2020 by the following grants: POCI01-0145-FEDER-007672, POCI-01-0145-FEDER-016880, POCI-01-0145-FEDER016886, POCI-01-0145-FEDER-028953, POCI-01-0145-FEDER-032113, PTDC/FISAST/1526/2014, PTDC/FIS-AST/7073/2014, \& UID/FIS/04434/2013. J.J.N. acknowledges support from FCT though the PhD::Space fellowship PD/BD/52700/2014.

\section{References}

Artigau, É., Kouach, D., Donati, J.-F., Doyon, R., Delfosse, X., Baratchart, S., Lacombe, M., et al. (2014). SPIRou: The near-infrared spectropolarimeter/high-precision velocimeter for the Canada-France-Hawaii telescope. In Proc.SPIE (Vol. 9147, p. 914715). International Society for Optics and Photonics. doi:10.1117/12.2055663

Artigau, É., Malo, L., Doyon, R., Figueira, P., Delfosse, X., \& Astudillo-Defru, N. (2018). Optical and Near-infrared Radial Velocity Content of M Dwarfs: Testing Models with Barnard's Star. The Astronomical Journal, 155, 198. doi:10.3847/1538-3881/aab77d 
Astropy Collaboration, Robitaille, T. P., Tollerud, E. J., Greenfield, P., Droettboom, M., Bray, E., Aldcroft, T., et al. (2013). Astropy: A community Python package for astronomy, 558, A33. doi:10.1051/0004-6361/201322068

Baraffe, I., Homeier, D., Allard, F., \& Chabrier, G. (2015). New evolutionary models for pre-main sequence and main sequence low-mass stars down to the hydrogen-burning limit. Astronomy and Astrophysics, 577, A42. doi:10.1051/0004-6361/201425481

Bouchy, F., Doyon, R., Artigau, É., Melo, C., Hernandez, O., Wildi, F., Delfosse, X., et al. (2017). Near-InfraRed Planet Searcher to Join HARPS on the ESO 3.6-metre Telescope. The Messenger, 169, 21-27. doi:10.18727/0722-6691/5034

Bouchy, F., Pepe, F., \& Queloz, D. (2001). Fundamental photon noise limit to radial velocity measurements. Astronomy and Astrophysics, 374(2), 733-739. doi:10.1051/ 0004-6361:20010730

Connes, P. (1985). Absolute astronomical accelerometry. Astrophysics and Space Science, 110(2), 211-255. doi:10.1007/BF00653671

Czekala, I., Andrews, S. M., Mandel, K. S., Hogg, D. W., \& Green, G. M. (2015). Constructing A Flexible Likelihood Function For Spectroscopic Inference. The Astrophysical Journal, 812(2), 128. doi:10.1088/0004-637x/812/2/128

Figueira, P., Adibekyan, V. Z., Oshagh, M., Neal, J. J., Rojas-Ayala, B., Lovis, C., Melo, C., et al. (2016). Radial velocity information content of $M$ dwarf spectra in the nearinfrared. Astronomy and Astrophysics, 586, A101. doi:10.1051/0004-6361/201526900

Gray, D. F. (2005). The Observation and Analysis of Stellar Photospheres (3rd ed.). Cambridge University Press. doi:10.1017/CBO9781316036570

Hunter, J. D. (2007). Matplotlib: A 2D graphics environment. Computing In Science 6 Engineering, 9(3), 90-95. doi:10.1109/MCSE.2007.55

Husser, T.-O., von Berg, S. W., Dreizler, S., Homeier, D., Reiners, A., Barman, T., \& Hauschildt, P. H. (2013). A new extensive library of PHOENIX stellar atmospheres and synthetic spectra. Astronomy and Astrophysics, 553, A6. doi:10.1051/0004-6361/ 201219058

Joblib: Running Python functions as pipeline jobs — joblib 0.13.2 documentation. (n.d.). Retrieved February 19, 2019, from https://joblib.readthedocs.io/en/latest/\#

Jones, E., Oliphant, T., Peterson, P., \& others. (2001-2001--). SciPy: Open source scientific tools for Python. Retrieved from http://www.scipy.org/

McKinney, W. (2010). Data Structures for Statistical Computing in Python. In (pp. 51-56). Presented at the Proceedings of the 9th Python in Science Conference. Retrieved from http://conference.scipy.org/proceedings/scipy2010/mckinney.html

Oliphant, T. E. (2015). Guide to NumPy (2nd ed.). USA: CreateSpace Independent Publishing Platform.

Pepe, F., Molaro, P., Cristiani, S., Rebolo, R., Santos, N. C., Dekker, H., Mégevand, D., et al. (2014). ESPRESSO: The next European exoplanet hunter. Astronomische Nachrichten, 335(1), 8. doi:10.1002/asna.201312004

Price-Whelan, A. M., Sipőcz, B. M., Günther, H. M., Lim, P. L., Crawford, S. M., Conseil, S., Shupe, D. L., et al. (2018). The Astropy Project: Building an Open-science Project and Status of the v2.0 Core Package, 156, 123. doi:10.3847/1538-3881/aabc4f

Reiners, A., Zechmeister, M., Caballero, J. A., Ribas, I., Morales, J. C., Jeffers, S. V., Schöfer, P., et al. (2018). The CARMENES search for exoplanets around M dwarfs. High-resolution optical and near-infrared spectroscopy of 324 survey stars. Astronomy and Astrophysics, 612, A49. doi:10.1051/0004-6361/201732054 
Tqdm/tqdm: Tqdm v4.31.1 stable | Zenodo. (n.d.). Retrieved February 19, 2019, from https://zenodo.org/record/2561403\#.XGtaB7iYZdg 